\title{
Reuse of ground tire rubber (GTR) as a filler of TPE matrix ${ }^{*}$
}

\author{
Kinga M ajewska-Laks ${ }^{1, * *}$, Dariusz Sykutera ${ }^{1}$ and Adrian Ościak $^{1}$ \\ 1UTP University of Science and Technology, Faculty of M echanical Engineering, Al. prof. S. \\ K aliskiego 7, 85-796 Bydgoszcz, Poland
}

\begin{abstract}
U sed car tires are the type of waste that does not decompose naturally and is not recyclable in molten state. Despite many initiatives to reuse these materials on a global scale, a large part of used tires are still landfilled. The present work is an attempt to use ground powder of ground tire rubber (GTR) to filling the TPE-S thermoplastic elastomer matrix. The aim of this study was to obtain TPE-GTR compositions in standard thermoplastic processing machines. This study used rubber powder with a grain size of $0.2-0.4 \mathrm{~mm}$, obtained by grinding process in a knife mill of own design. The GTR filler into the TPE matrix in the amount of 5 and 50 wt $\%$ was dosed. Specimens were produced by injection molding. The obtained molded parts were tested for tensile strength, Shore hardness and Schob elasticity. Influence of melt temperature on TPE -GTR molded parts properties was also examined. The addition of GTR to the matrix causes a significant decrease in elongation at break, while improving the stiffness, damping properties and hardness of the TPE-GTR composition. It was also found that the melting temperature above $200^{\circ} \mathrm{C}$ has negative effect on the properties of the TPE-GTR compounds.
\end{abstract}

\section{Introduction}

The analysis of the current EU waste management legislation shows that for EU mechanical recycling is a primary technology for the management of used car tires. One of the ways of recycling rubber from car tires is its grinding to rubber granulates (Ground Tire Rubber GTR) and use as a filler for thermoplastics. Thermoplast-GTR products are made by standard thermoplastic processing such as injection molding and extrusion [1-3].

The compounds properties are determined by the type of matrix, the filling degree and the size distribution of the filler grains as well as their unifom distribution in the thermoplastic matrix [1,2]. The biggest research challenge is increasing GTR grains adhesion to the matrix. Therefore, many attempts are made to preparation the rubber filler by modifying the grain surface or using effective compatibilizer additives $[4,5]$.

The use of GTR powder as a filler causes decrease of the mechanical properties of the compounds in relation to the matrix material. Optimal processing conditions for thermoplastics/GTR compounds are searched for, in the aspect a utility properties increase of this material [2].

Formela found that during the recycling of vulcanized rubber there was a weight loss due to thermal decomposition. It was the lowest during GTR extrusion at the plasticizing temperature of $180^{\circ} \mathrm{C}(0.57 \mathrm{wt} \%)$ and the highest at $280^{\circ} \mathrm{C}(4.46 \mathrm{wt} \%)$ [6].

A beneficial effect on the mechanical properties of composites with GTR grains was found in the case of GTR regeneration using microwaves irradiation. The de-vulcanization

* This work was supported by the and Polish $N$ ational Center for R esearch and Development within the framework of the funding initiative EUREKA/TIREC/2/2019 (E!13042 TIREC)

** Corresponding author: Kinga.M ajewska-Laks@ utp.edu.pl 
carried out with this method had a positive effect on the adhesion of the thermosetting matrix to the filler [7].

Studies showed a significant influence of processing temperature, screw rotation speed and extrusion efficiency during the production of PEHD/GTR and PELD/GTR composites on their mechanical and rheological properties. The applied values of the melt temperature had a large impact on the melt flow rate, hardness, tensile strength and elongation at break of the obtained samples [8,9]. It was also found that the addition of maleic anhydride (MA) to PEHD-g-MAH polyethylene has a positive effect on the homogeneity of GTR dispersion in the polyolefin matrix and on interfacial interactions [9].

Sienkiewicz indicated the significant influence of the grain size and of the compatibility technique on the final properties of thermoplastic/GTR compound. Ground Rubber added to the PP matrix causes increase in the elongation at break and an increase in PP impact strength, already at $20 \mathrm{wt} \%$ of filling degree. The reason for the poor mechanical properties of the discussed composites is the low interfacial interaction between the filler and the matrix. It was suggested to use compatibilizers as adhesion promoters and as an antiagglomerating agents in the compound. The improvement of the mechanical properties was achieved by the prepare a compound consisting of PP grafted with maleic anhydride (MA), GTR and SEBS also grafted with MA $[1,2]$. Importantly, the surface of GTR grains has a significant influence on their adhesion to the matrix. It was found that rubber grains obtained at ambient temperature more porous and ragged surface were characterized, which slightly improves the impact strength of PELD/GTR compounds $[1,2,10]$.

According to the team led by Hrdlicek, the highest values of tensile strength and elongation at break are achieved by using the smallest possible GTR particles. In their research, they made LDPE/GTR composite supported by EPDM or SEBS. LDPE constituted $50 \%$ of the composite, GTR - 25\%, while EPDM and SEBS were used interchangeably in the amount of $25 \%$. The GTR grain size was split into six fractions from $0.12 \mathrm{~mm}$ to $0.65 \mathrm{~mm}$. The best results were achieved with the use of GTR with a fraction of $0.12 \mathrm{~mm}$ and the use of SEBS or EPDM as a compatibilizer [11]. These results were confirmed for PP/GTR compound. A decrease in the mechanical properties of composites was observed with an increase in particle size and with an increasing degree of compound filling [12]. Another compatibilizer improving the mechanical properties of PE/GTR and PP/GTR composites can be a coupling agent containing silanes or sulfuric acid. The use of these agents has a very positive effect on the increase of crystallinity of matrix materials. This observed effect together with growth the specific surface of rubber grains improvement the mechanical properties of compound was caused [13, 14].

There is also an example of TPE-U/GTR composite reinforced with kenaf fibers. The kenaf content in the composite was $12.5 \%$ and caused an increase in tensile and flexural strength and an increase in impact strength [15]. Another example of a three-component composite is PE filled with talc or wood flour and GTR. Such a composite is characterized by an increased modulus of elasticity, higher value of density and hardness. Additionally, the presence of wood flour reduces the thermal stability of the composite [16].

Tests showed, that PP/GTR compounds an excellent sound absorption were characterized. In the grain size range from $0.8 \mathrm{~mm}$ to $2.0 \mathrm{~mm}$, the best sound absorption the GTR-filled compound with a grain size of $2.0 \mathrm{~mm}$ was showed. It was prepared in the proportion of 50:50, without any additives [17]. In other studies it was found that for of PEHD/GTR compound, the optimal acoustic attenuation was observed for molded parts with $20 \mathrm{wt} \%$ GTR content. At the same time, for the high rubber filled compound, decrease tensile modulus and increase crystallinity degree of matrix was observed [18].

He Yue's et al. found that the added of GTR grains to cellular PP matrix improved its cell structure and improved a homogeneously distributed fine pores. The obtained PP/PP- 
$\mathrm{MAH} / \mathrm{CNBR}$ compound the impact strength of about 2.2 times higher than that of solid PP was characterized. Simultaneous decrease in tensile strength by $26 \%$ was observed [19].

Currently, several directions of composite modification based on GTR filling are being investigated in order to find their mass applications. Among others, the compatibility of the thermoplastic matrix to GTR grains is of great interest to researchers. A completely different approach is reinforcing of GTR compounds with fibers and exploration new applications that do not require high mechanical properties, for example dielectric materials used in electronics [4, 20, 21].

The aim of the study was to determine the influence of a GTR content and filler grain size on the mechanical properties of TPE-GTR compound. Size range 0.2-0.4 mm rubber grains were used as TPE filler. The properties of the TPE compound filled with GTR grains have not been published so far. Expected that, the final ground rubber content in the TPE will be comparable to the volume matrix content and the samples will be made using standard thermoplastics processing.

\section{Methodology}

TPE-S thermoplastic elastomer from the FC series with the trade name TF2CGT (Kraiburg TPE, Germany) as a matrix was used. This material by a hardness of 18 ShA, a tensile strength of $5 \mathrm{MPa}$ and an elongation at break of $800 \%$ was characterized [22].

The thermoplastic elastomer was filled with GTR tire rubber powder, which was obtained in a knife mill with a own special design [23]. Tire rubber granules with a grain size of about $7 \mathrm{~mm}$ finely were grounded to a grain size of $0.2-0.4 \mathrm{~mm}$ by the hyperboloid cutting. This method in previous publications was described [23-25]. The rubber grains obtained by knife cutting were characterized by flat surfaces and sharp edges (Fig. 1). That means that the main reason for volume reduction of the feeded elastomeric material was cutting with rectilinear knife edges in the gap between knifes with the value $0.1 \mathrm{~mm}$. The rubber powder was added to the TPE matrix in the amount of 5 and $50 \mathrm{wt} \%$.

a)

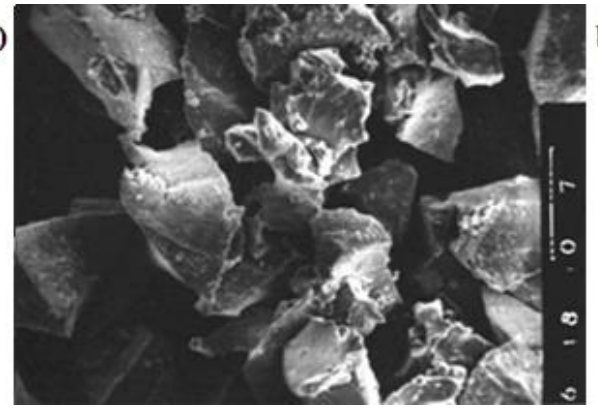

b)

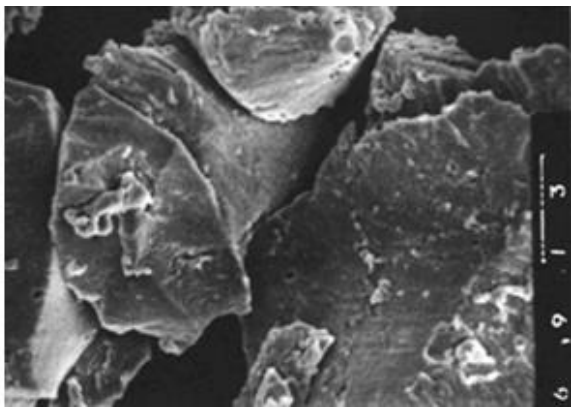

Fig. 1. SEM images of GTR rubber grains obtained by grinding in the UTP knife mill: a) magnification $\mathrm{x} 100, \mathrm{~b})$ magnification $\mathrm{x} 300$ [25].

Fusabond M603 (Dow Corporate, USA) as compatibilizer improving the adhesion between the matrix material and the rubber grains was used [26]. It was added to the TPE$\mathrm{S}$ matrix in the amount of $3 \mathrm{wt} \%$. TPE-S granules were dried in the Binder FED1 15 thermal chamber at $80^{\circ} \mathrm{C}$ for 4 hours.

In the first stage, TPE-S/GTR compounds by a laboratory single-screw extruder W2530D (Metalchem, Poland) with a screw diameter of $25 \mathrm{~mm}$ and a length to diameter ratio of 30 were prepared. During extrusion, the screw rotation speed was $50 \mathrm{rpm}$, and feed rate 8 $\mathrm{m} / \mathrm{min}$ was calculated. The temperature of individual heating zones of the extruder plasticizing system respectively to $140,160,170^{\circ} \mathrm{C}$ and $170^{\circ} \mathrm{C}$ (extrusion head) were set.

The obtained TPE/GTR extrudates with a C13.20SV low-speed knife mill (Wanner Technik GmbH, Germany) were ground. The rotor with 9 movable knives arranged in 3 
segments and 2 fixed knives was equipped. During grinding, a sieve with a hole diameter of $5 \mathrm{~mm}$ was used and the inter-knife gap was $0.1 \mathrm{~mm}$.

In the last stage, the TPE/GTR molded parts by the injection molding method using the Engel E-Victory 110 injection molding machine with a maximum clamping force of 1100 $\mathrm{kN}$, equipped with a $35 \mathrm{~mm}$ diameter screw were obtained. Specimens type A1 in accordance with PN-EN ISO 527-2 in the four-cavity laboratory injection mold (UTP, Poland) were made. The tool with the M10 chiller (TAEevo, Italy) and the HB - 160Z1 thermostat (HB - THERM, Switzerland) was heated. The constant parameters of the injection process were: flow rate $40 \mathrm{~cm}^{3} / \mathrm{s}$, the holding time $10 \mathrm{~s}$, the cooling time $20 \mathrm{~s}$. The variable factors in the experiment carried out were: injection mold temperature and temperature profile of plasticizing zones (Table 1) and composition of TPE/GTR compounds (Table 2).

Table 1. Variants of temperature settings adopted in the manufacturing process of TPE/GTR compounds.

\begin{tabular}{|c|c|c|}
\hline Heating zone & Temperature A, $^{\circ} \mathbf{C}$ & Temparature $\mathbf{B},{ }^{\circ} \mathbf{C}$ \\
\hline Feeding zone & 140 & 190 \\
\hline Compression zone & 160 & 210 \\
\hline Mettering zone & 170 & 230 \\
\hline Injection nozzle & 170 & 230 \\
\hline Injection mold & 25 & 50 \\
\hline
\end{tabular}

Table 2. Composition of TPE/GTR compounds with the variants of temperature settings realized in injection molding process.

\begin{tabular}{|c|c|c|c|}
\hline Specimen & TPE-S/GTR & $\begin{array}{c}\text { Compatibilizer content, } \\
\mathbf{w t} \%\end{array}$ & $\begin{array}{c}\text { Variant of temperature } \\
\text { settings }\end{array}$ \\
\hline TPS & $100: 0$ & 0 & $\mathrm{~A}$ \\
\hline TPSHT & $100: 0$ & 0 & $\mathrm{~B}$ \\
\hline TPS/GTR5 & $95: 5$ & 0 & $\mathrm{~A}$ \\
\hline TPS/GTR5K & $95: 5$ & 3 & $\mathrm{~A}$ \\
\hline TPS/GTR50 & $50: 50$ & 0 & $\mathrm{~A}$ \\
\hline TPS/GTR50HT & $50: 50$ & 0 & $\mathrm{~B}$ \\
\hline TPS/GTR50K & $50: 50$ & 3 & $\mathrm{~A}$ \\
\hline
\end{tabular}

The adopted temperature values were based on data provided by the material manufacturer within the range of recommended TPE processing parameters. Additionally, thermogravimetric curves (TG), which were carried out in a nitrogen atmosphere using the NETZSCH TG 209F1 Libra thermogravimeter with a heating rate of $10^{\circ} \mathrm{C} / \mathrm{min}$, in the temperature range from room temperature up to $1000^{\circ} \mathrm{C}$ were analyzed. All components of the compound were assessed based on $10 \mathrm{mg}$ weight samples.

The tensile strength of the obtained molded parts was determined using the Z030 testing machine (Zwick \& Roell, Germany), equipped with the TestXpert II control program. Measurements of 5 samples of each material group were made in accordance with PN-EN ISO 527, using a tensile speed of $50 \mathrm{~mm} / \mathrm{min}$. In the paper were marked: $E_{t}[\mathrm{MPa}]-$ Young's modulus; $\sigma_{\mathrm{m}}[\mathrm{MPa}]$ - tensile strength; $\varepsilon_{\mathrm{m}}[\%]$ - elongation at break. 
The hardness determination was carried out using the HPE II durometer (Zwick \& Roell, Germany). Measurements in accordance with the PN - EN ISO868: 2005 standard at 10 points in the sample symmetry axis and in the direction of the material flow in the injection mold cavity were made.

The elasticity of the samples using Schob method with EPGi 772 apparatus (Fritz Heckert, Germany), in accordance with PN - C 04255: 1997 was determined. The samples were cut from the part of the test piece at the end of the flow path and then 3 pieces were stacked.

\section{Results}

Based on the analysis of the thermomechanical TG curves, it can be observed that the least thermally resistant component of the TPE-S/GTR composition is tire rubber powder (Fig. 2). At $95^{\circ} \mathrm{C}$, the weight loss was $0.5 \%$ by mass, while a $1 \%$ reduction at $166^{\circ} \mathrm{C}$ was observed. TPE-S was more thermally resistant, with weight losses of $0.5 \%$ by mass and $1 \%$ by mass observed at 181.2 and $232^{\circ} \mathrm{C}$, respectively. Against the background of weight changes of the main components of the TPE-S/GTR composition, the Fusabond M603 compatibilizer is the most thermally stable.

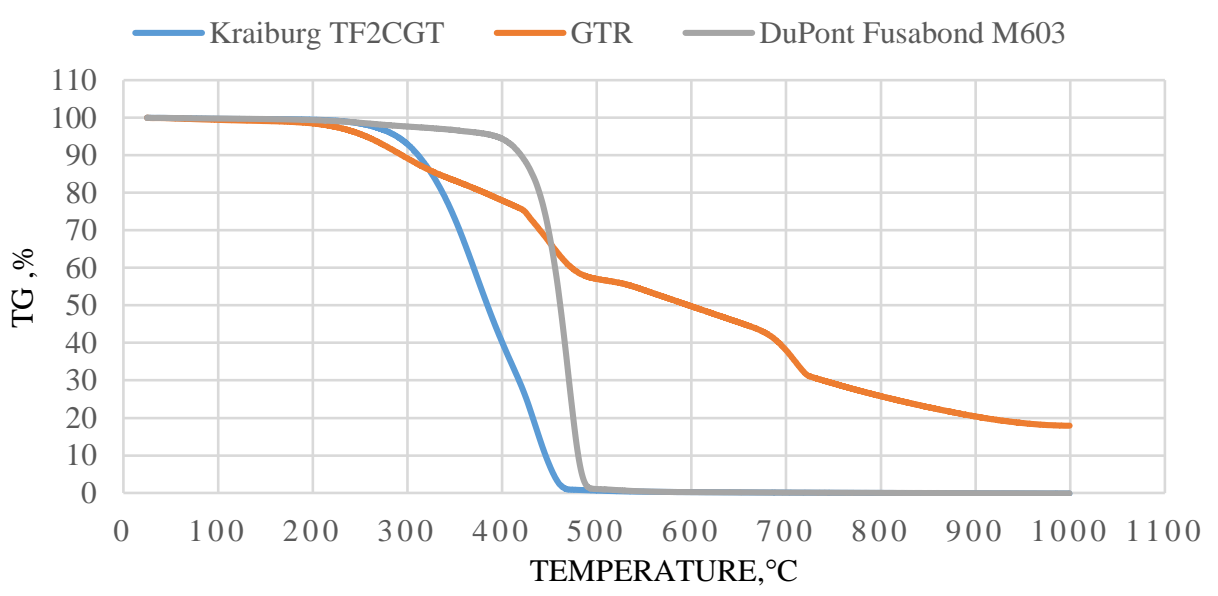

Fig. 2. TG curves for all components contained in the TPE-S/GTR compound.

The significant influence of the time and of the temperature on the rubber powder bechaviour on the TG curves determined in the simulation of process conditions was noticeable (Fig. 3). Keeping GTR at $180^{\circ} \mathrm{C}$ for 16 minutes caused a decrease in its mass by $1.46 \%$, and at $250^{\circ} \mathrm{C}$ by over $9 \%$. Effect of the initiation of the vulcanized rubber degradation process at temperature above $200^{\circ} \mathrm{C}$ was observed. The presented thermogravimetric analyzes were the basis for settings favorable processing temperature for both the extrusion and injection molding process. This way, the possible occurrence of degradation of rubber grains in both processes by reducing the plasticization temperature and the residence time of the components in the plasticizing system was minimized. 


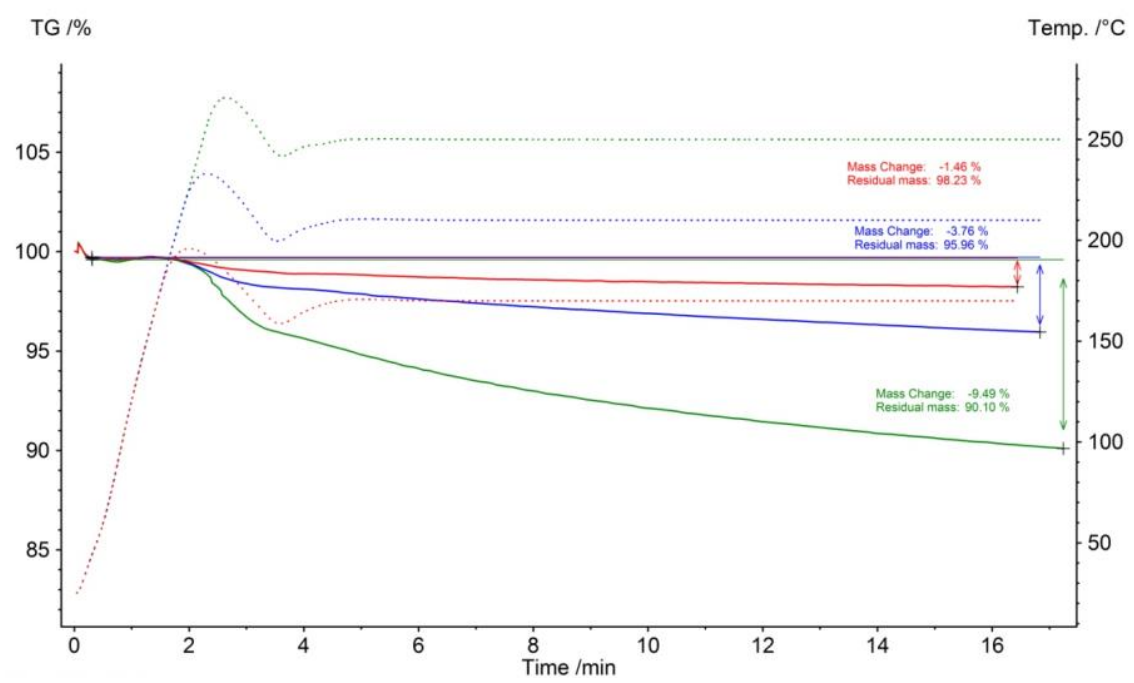

Fig. 3. The effect of the high temperature exposure time on the weight decrease of the tire rubber powder contained in the TPE-S/GTR compound.

Filling the thermoplastic elastomer matrix with rubber powder reduced the elongation of the TPE-S/GTR compound. It was especially noticeable for the material containing $50 \mathrm{wt} \%$ rubber powder (Fig. 4). The tensile strength $R_{m}$ was significantly reduced even when the compound with a rubber powder in the amount of 5\% by weight was filled. Increasing the GTR content to $50 \mathrm{wt} \%$ caused slight increase of compound elasticity. This is a opposite effect compared to the results of the modification of polyolefin materials, polyamide or polystyrene with rubber powder. Also was a significant increase in $R_{m}$ and Young's modulus for the compound containing the compatibilizer (Fig. 4-5). Particularly interesting effects were obtained for high filled. Amount $50 \mathrm{wt} \%$ of rubber filler in the TPE matrix caused a significant increase in the modulus of elasticity, also for compounds without a compatibilizer. In the case of the TPE-S/GTR50K compound, the tensile strength was kept similar to the $\mathrm{R}_{\mathrm{m}}$ value for TPE-S (Fig. 5). It was also observed that despite the significant rubber powder content, the decrease in the elongation of the compound was not as significant as in the case of rubber filled polyolefins.

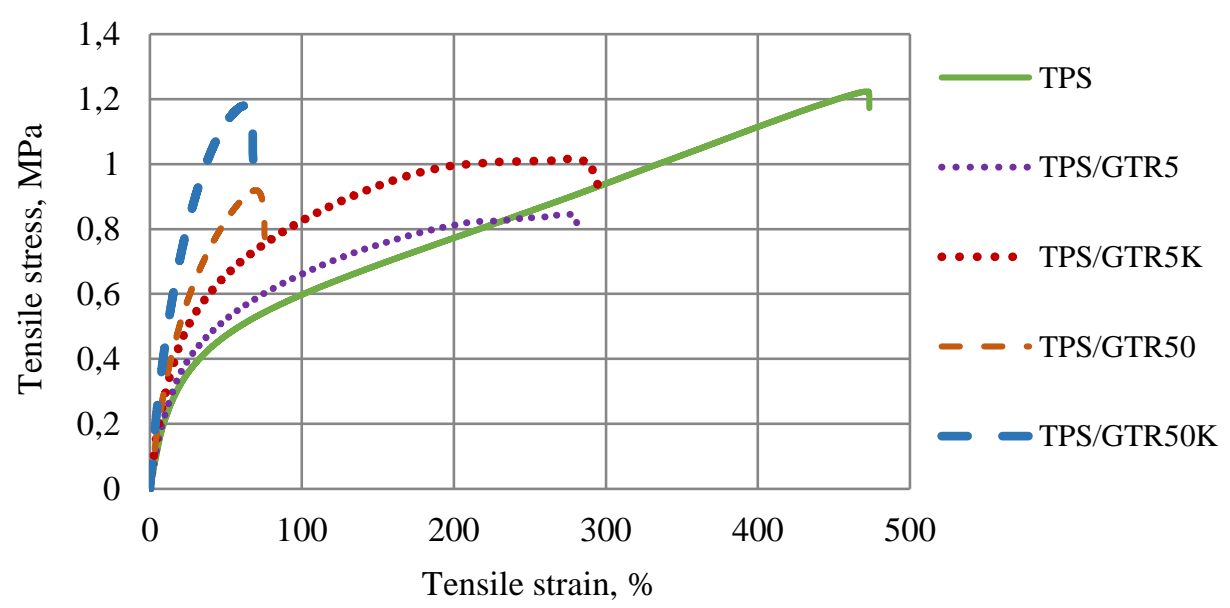

Fig. 4. Tensile curves for the TPE-S/GTR compounds. 


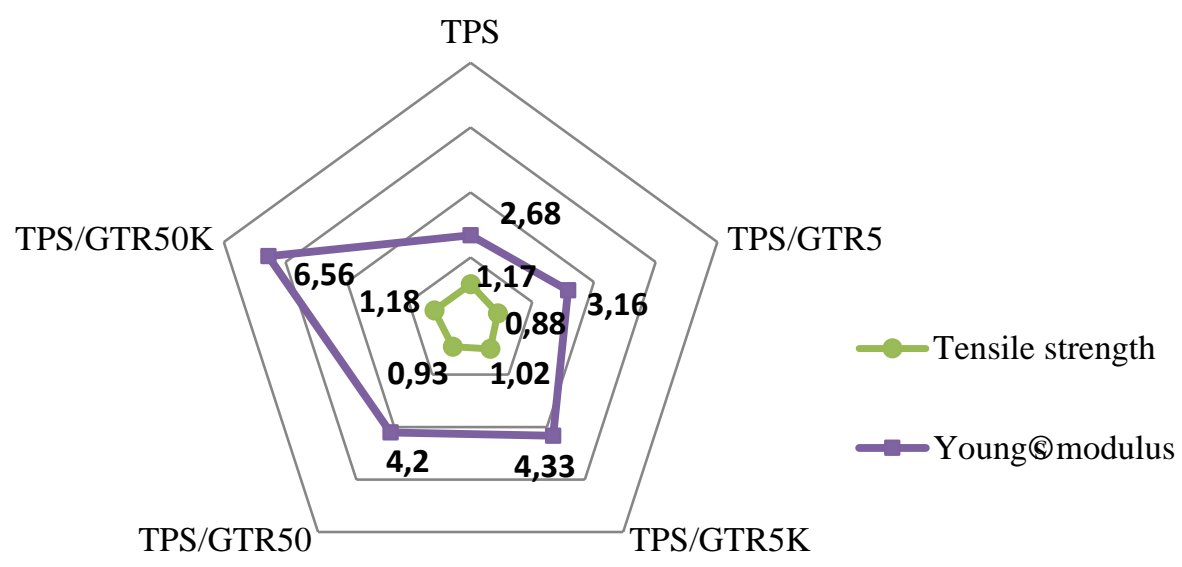

Fig. 5. Changes in the mechanical properties of TPE-S/GTR compounds depending on the content of rubber powder and compatibilizer.

These types of different behaviour of compound caused from the fact that the TPE matrix is characterized by low hardness, it is flexible, and its tensile strength is about 1 $\mathrm{MPa}$. The changes in the mechanical properties of the composition resulting from the simultaneous addition of a large amount of rubber powder and of the compatibilizer were different from the effects described in the literature.

In the case of polypropylene and polyamide, an increase in the rubber powder content caused a significant decrease in tensile strength. The observed reinforcement of the TPE matrix was also reflected in the increase in the hardness of the compound (Fig. 6), the most important in the case of the simultaneous use of a compatibilizer and the $5 \%$ GTR content in the compound. High standard deviation of this parameter was not observed, even in the case of a high content of rubber powder. This is an indirect confirmation that homogeneous compound were prepared.

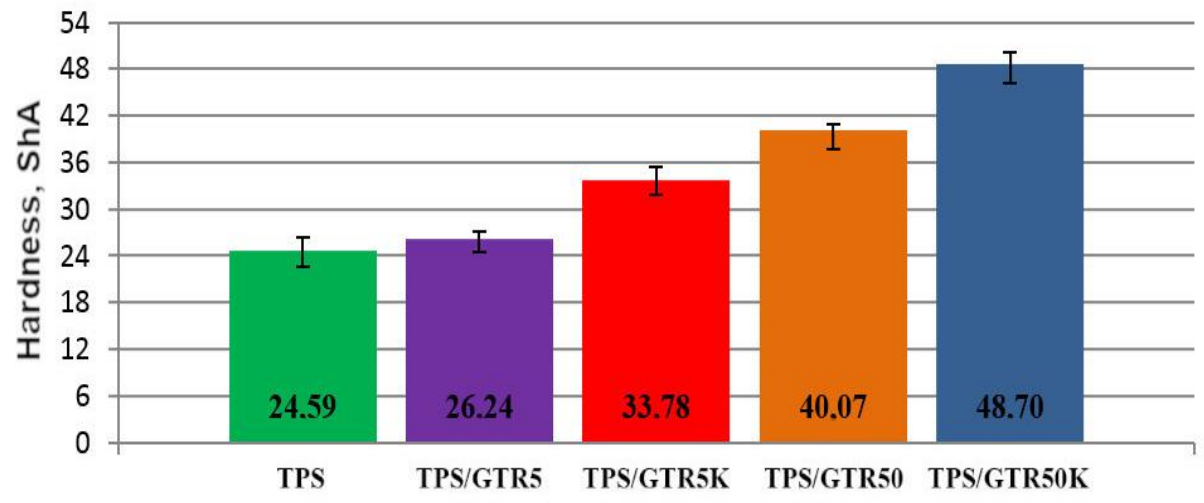

Fig. 6. Influence of ground rubber content and of compatibilization process on the hardness of the TPE-S/GTR compounds.

The results obtained in the flexibility test were consistent with the literature data on the modification of polyolefin and construction materials. The addition of rubber powder to the thermoplastic matrix usually causes a significant decrease in elasticity and an increase in damping properties. A significant portion of the mechanical energy acting on the sample was dissipated by the composition structure at the matrix/GTR interface and by the rubber 
filler itself. In the TPE-S/GTR specimens tested, a decrease in elasticity was observed, but not as significant as in compositions with harder thermoplastic matrices. The reason for the observed changes was the flexibility of both components of the mixture and their mutual positive interaction, resulting in a slight decrease in flexibility (Fig. 7).

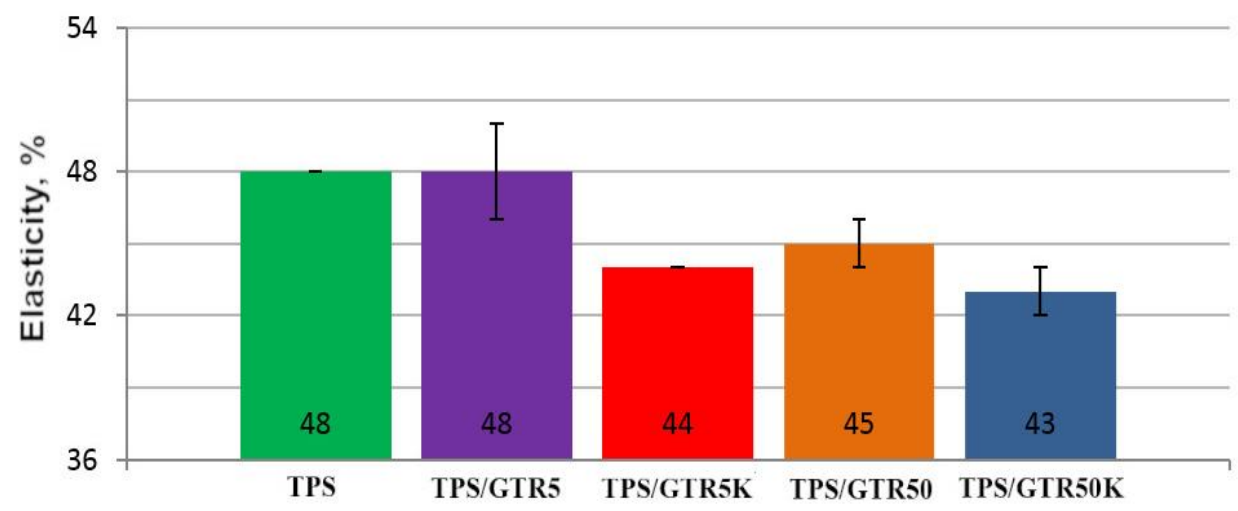

Fig. 7. Influence of ground rubber content and of compatibilization process on the elasticity of the TPE-S/GTR compounds.

The significant influence of injection mold and plasticizing temperature on the strength properties of TPE-S and the TPE-S/GTR compound was observed (Fig. 8). Pure TPE-S samples were characterized by a significant increase in tensile strength by $224 \%$, while maintaining high flexibility and reducing Young's modulus by about $50 \%$. The increase in the melting temperature of the TPE-S/GTR compound and its injection into the warmer mold cavity caused a significant reduction of $R_{m}$ by $72 \%$, as well as a significant decrease of the elasticity modulus to the value characteristic for samples obtained from TPS (a decrease by $36 \%$ ).

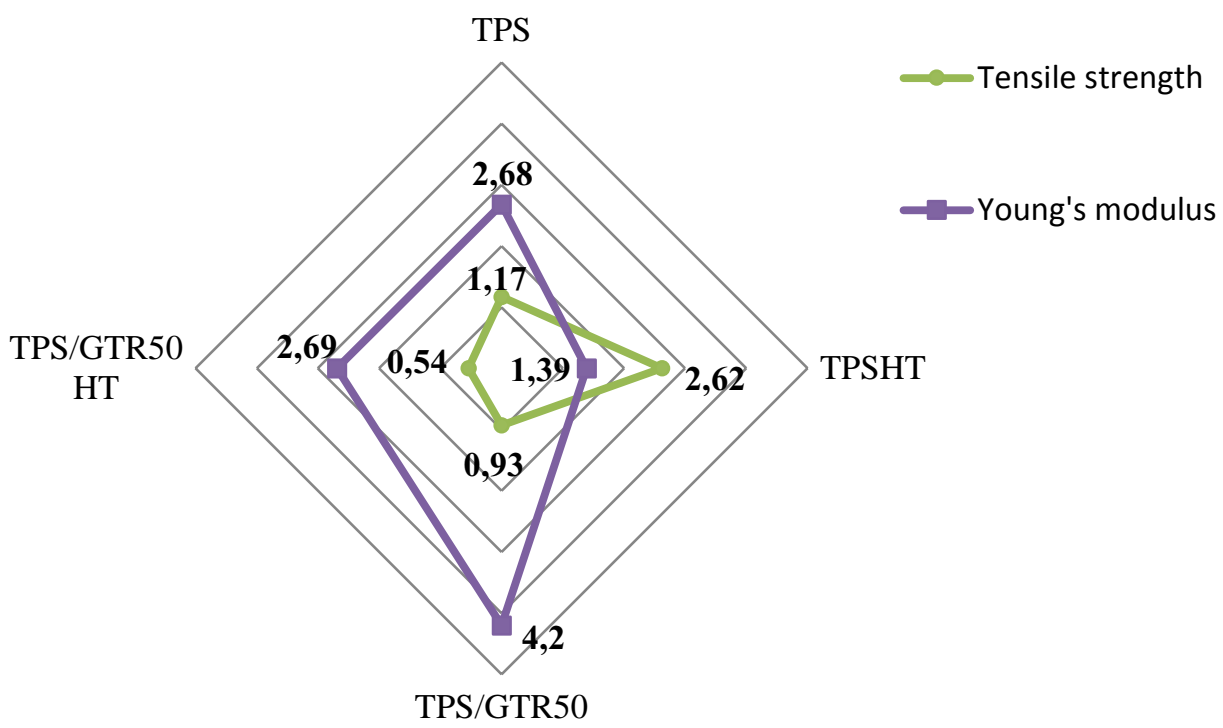

Fig. 8. Influence of the high temperature in the plasticizing unit of the injection molding machine and in the mold cavity on the tensile strength and Young's modulus for TPE-S and TPE-S/GTR molded parts. 


\section{Conclusions}

Based on obtained results can be conclude that the addition of GTR to the TPE-S matrix causes a significant decrease in elongation at break, while improving the stiffness and hardness of the compounds. The compatibilizer further improves these parameters by promoting the interfacial interaction between the cross-linked rubber grains and the polymer matrix. With the use of GTR filling at the level of $50 \%$ and the addition of a compatibilizer, the tensile strength is maintained at a similar level to TPE-S, and the modulus of elasticity has increased more than twice. The addition of a compatibilizer and an increase in the GTR content reduce the flexibility of the composite and improve its damping properties. This study also shows that the processing parameters have a important influence on the mechanical properties of the compound. Temperature that are too high cause the small grains of rubber from car tires to undergo irreversible decomposition processes, which affects its adhesion to the matrix and lower the properties of the compound. In order to obtain the optimal properties of compounds filled with ground tire rubber, a matrix with the lowest possible processing temperature should be used. This will minimize the occurrence of degradation of the rubber grains.

\section{References}

1. J. Sienkiewicz, H. Janik, K. Borzędzkowska-Labuda, J. Kucińska-Lipka, J. Clean. Prod. 147, p.560-571 (2017)

2. M. Sienkiewicza, PhD Thesis, Gdańsk University of Technology (2010)

3. P. Lima, S. P. Magalhaes da Silva, J. Oliveira, V. Costa, Polym. Test. 45, p.58-67 (2015)

4. J. Karger-Kocsis, L. M eszaros, T. Barany, J. M ater. Sci. 48, p.1-38 (2013)

5. A. Hejna, M. K lein, M. Saeb, K. Formela, Polym. Test. 73, p.143-151 (2019)

6. K. Formela, M. Bogucki, P. Stączek, Polimery, 60, p.57-65 (2015)

7. K. A oudia, S. Azem, N. A it Hocine, M. Gratton, V. Pettarin, S. Seghar, Waste M anagement, 60, p.471-481 (2017)

8. K. Formela, M. B ogucki, Polimery, 59, p.488-494 (2014)

9. P. Song, S. Li, S. Wang, Polym. Degrad. Stabil. 143, p.85-94 (2017)

10. M. Bekhiti, H. Trouzione, A. A sroun, Eng. Technol. A ppl. Sci. Res. 4, 4, p. 669-672 (2014)

11. Z. Hrdlicka, P. M. Marin Cebria, V. Stefan, A. Kuta, Prog. Rubber Plast. Recycl. Technol. 32, 3, p. 129-141 (2016)

12. E. Frida, M akara J. Technol. 16, 2, p.171-179 (2012)

13. S. Satapathya, A. Nagb, G. B. Nandoa, Process Saf. Environ. Prot. 88, p. 131-141 (2010)

14. E. Hernandez, J. F. Hernandez Gomez, L. F. Cepeda, E. J. Chavez M unoz, F. Soriano Corral, S. G. Solıs Rosales, G. Neira Velazquez, P. Gonzalez Morones, D. I. Sanchez Martınez, J. A ppl. Polym. Sci. 134, 21 (2017)

15. A.M. Noor A zammi, S.M. Sapuan, M.R. Ishak, M.T.H. Sultan, Fibers and Polym. 19, p. $446-451$ (2018)

16. A. Ramezani Kakroodi, D. Rodrigue, J. A ppl. Polym. Sci. 131, 8 (2014)

17. J. Zimniak, B. Królikowski, Inż. A par. Chem. 53, p.396-397 (2014)

18. B. Haworth, D. Chadwick, L. Chen, Y.J. Ang, J. Thermoplast. Compos. M ater. 31, 1, p. 199-142 (2018)

19. Y. He, T. Jiang, Y. Liu, W. Gong, L. He, J. Mater. Eng. Perform. 45, 2, p. 80-87 (2017)

20. R. Mujal-Rosas, J. Orrit-Prat, X. Ramis-Juan, M. Marin-Genesca, A. Rahhali, Polym. Polym. Compos. 20, 9, p. 797-808 (2012)

21. L. K roll, S. Hoyer, M. K laerner, Procedia M anufacturing, 21, p. 591-598 (2018) 
22. K raiburg TPE TF2CGT (FC series) Technical Data Sheet (2018)

23. R. Konieczka, W. Kałużny, D. Sykutera, Kautsch. Gummi K unstst. 9, 50 (1997)

24. S. Hoyer, L. K roll, D. Sykutera, Procedia M anufacturing, 43, p. 193-200 (2020)

25. D. Sykutera, Dissertation N o. 169, Wyd. UTP, Bydgoszcz (2013)

26. DuPont Fusabond M 603 Product Data Sheet (2014) 